\section{Infliximab is ineffective against polymyalgia rheumatica}

Treatment with glucocorticoids can resolve most cases of polymyalgia rheumatica within 2 years; however, a minority of patients have a chronic, relapsing form of the disease and require long-term glucocorticoid treatment, which carries a high risk of steroid-related adverse events. After a pilot study suggested that infliximab might have a steroid-sparing effect, Salvarani et al. evaluated the effect of infliximab in patients newly diagnosed with polymyalgia rheumatica in a randomized double-blind placebo-controlled trial. Results showed, however, that infliximab was no better than placebo as treatment for the condition.

Of the 51 patients enrolled, 23 received infliximab $(3 \mathrm{mg} / \mathrm{kg}$ at weeks $0,2,6,14$ and 22) and 28 received placebo. All patients also received $15 \mathrm{mg}$ prednisone daily for the first 4 weeks; the dose was then tapered to $2.5 \mathrm{mg}$ daily over the following 12 weeks. Two patients dropped out in the infliximab group because of adverse events - one because of giant cell arteritis and one because of systemic infection. Four patients in the infliximab group had infusion reactions, but treatment did not need to be discontinued. At 52 weeks, 6 patients (30\%) in the infliximab group and 10 patients $(37 \%)$ in the placebo group were free of relapse or recurrence. Limitations of the study included a low dose of infliximab, rapid prednisone tapering (which might have contributed to the high rate of disease flare-ups) and a small sample size (a larger treatment effect was expected).

The authors conclude that infliximab is unlikely to benefit patients with polymyalgia rheumatica.

Original article Salvarani C et al. (2007) Infliximab plus prednisone or placebo plus prednisone for the initial treatment of polymyalgia rheumatica: a randomized trial. Ann Intern Med 146: 631-639

\section{Web-based strategies to improve osteoporosis care}

In order to improve the standard of care for patients with glucocorticoid-induced osteoporosis (GIOP), researchers at the University of Alabama have developed a web-based intervention consisting of three case-based modules dealing with the management of patients with GIOP, together with audit and feedback of physicians' management of such patients. Curtis et al. conducted a randomized controlled trial of the program, to see if the intervention increased rates of BMD testing and prescription of osteoporosis medication among participating physicians.

Glucocorticoid-prescribing physicians were identified from the database of a large managed care organization. Eligible physicians were recruited to participate in an online continuing medical education program. Control participants received text-based modules dealing with chronic illnesses other than osteoporosis. The intervention course was taken by 78 physicians, the control course by 75 . All three modules were completed by 27 (34.6\%) physicians in the intervention group and by $18(24.0 \%)$ control participants. Intention-to-treat analysis revealed no difference in the rates of BMD screening or osteoporosis medication prescribing between intervention and control participants. When only the participants who completed the courses were considered, however, analysis indicated that intervention physicians were more likely than control physicians to screen for and treat GIOP, although the results were only of borderline statistical significance.

Although the web-based intervention did not improve GIOP management, the authors suggest that greater exposure might produce a greater effect. Combination strategies that involve both patients and providers are being tested.

Original article Curtis JR et al. (2007) Challenges in improving the quality of osteoporosis care for long-term glucocorticoid users: a prospective randomized trial. Arch Intern Med 167: 591-596

\section{Knee inflammation at first presentation for RA is associated with a severe disease course}

Although it is important to be able to identify patients who will experience a severe course of rheumatoid arthritis (RA), because these patients would benefit from early, aggressive treatment, prognostic methods to date have not yet achieved this goal. Linn-Rasker et al. investigated the distribution of inflamed joints at first presentation for RA as a possible predictor of its subsequent severity. 